\title{
Hydrosol prepared from Rosa damascena is a candidate for therapeutic agents against superficial candidiasis
}

\author{
Naho Maruyama1,2, Shigeru Tansho-Nagakawa ${ }^{3}$, Miki Takahashi², Chizuru Miyazaki ${ }^{3}$ \\ Kazuyuki Shimomura ${ }^{4}$, Naoki Shimazaki ${ }^{4}$, Yasuo Ono ${ }^{3}$, and Shigeru Abe ${ }^{2,4}$
}

1 Faculty of Health and Medical Science, Teikyo Heisei University, 2 Teikyo University Institute of Medical Mycology, 3 Department of Microbiology and Immunology, Teikyo University School of Medicine, 4 Faculty of Medical Technology, Teikyo University

\section{Introduction}

Hydrosol obtained from the flowers of Rosa damascena (rose water) has been traditionally used as skin conditioners against troubles such as erythema, itchiness and swelling. One of the causes of these troubles is microbial infection. The treatment for infectious conditions usually focuses on antimicrobial effects. However, inflammatory symptoms accompanying infection are serious under clinical conditions and therefore suppression of inflammation is also very important. Although there are anecdotal evidences regarding the efficacy of rose water, there have been few experimental studies to examine its activities. Here, we investigated its effects on the growth of $C$. albicans and other microbes, and on the function of neutrophils which play a major role in the regulation of inflammatory reactions.

\section{Rose water}

Rose water was donated from Bulgaria Rose Japan Ltd.

Content of essential oil in rose water : $0.095 \%$

Chemical composition of essential oil

Phenylethyl alchol : $\quad 43.65 \%$

Citronellol :

Geraniol :

$18.24 \%$

$14.83 \%$

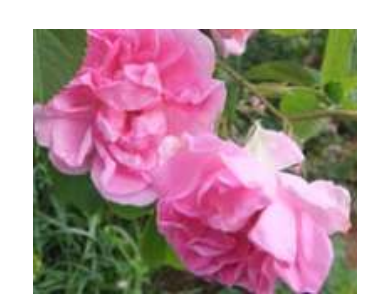

\section{Inhibitory effect of rose water on mycelial} growth of C. albicans a)

C. albicans were incubated with diluted rose water and its components for $16 \mathrm{~h}$ at $37^{\circ} \mathrm{C}$ in $5 \% \mathrm{CO}_{2}$. The mycelial growth of C. albicans was evaluated by measuring the $\mathrm{OD}_{620}$ of adherent mycelial cells to plastic plates.

Rose water inhibited mycelial growth of $C$. albicans at a concentration of ca. $2.2 \%$, and inhibited yeast growth at $50 \%$.

It was speculated that geraniol, and possibly citronellol, may contribute to the activity of rose water.

\section{Anti-microbial effect on other microbes}

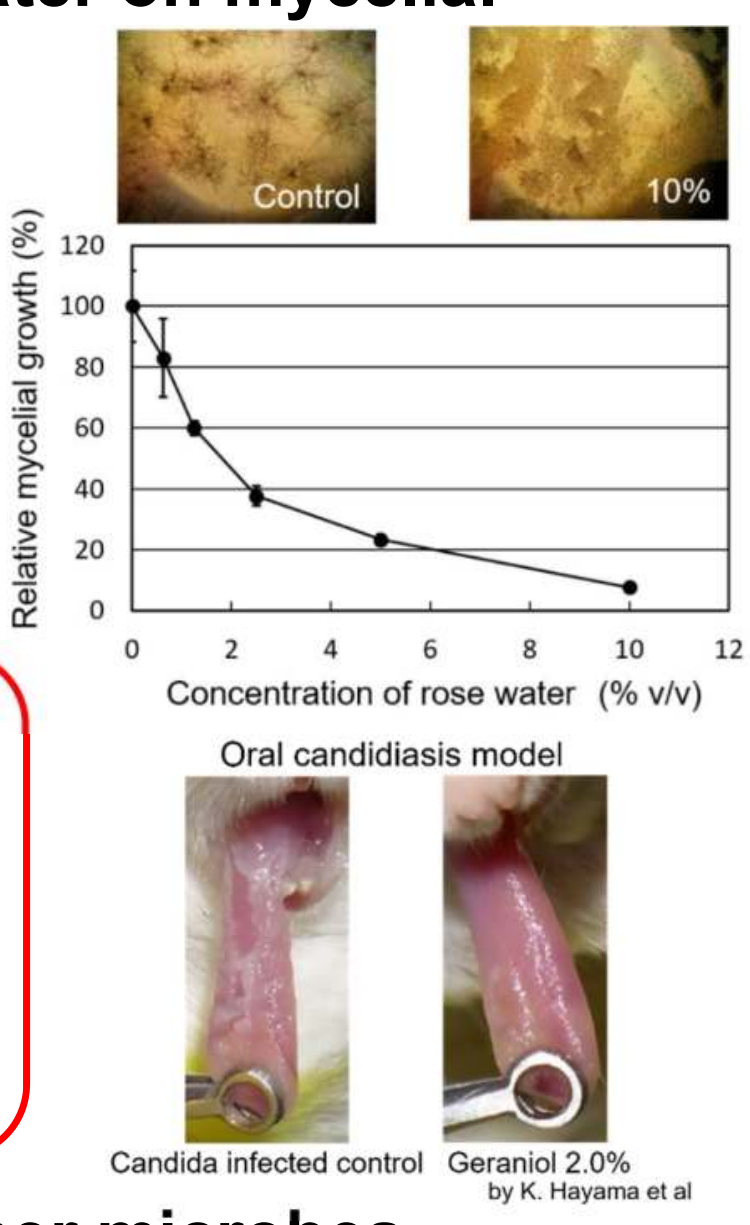

Rose water reduced viability of MRSA within $1 \mathrm{~h}$, and partially suppressed the growth of $M$. pachydermatis.

\section{Discussion}

From these results, it was indicated that rose water reduced the pathogenicity of candida by inhibiting its mycelial growth, inhibited the growth of MRSA and $M$. pachydermatis, and attenuated neutrophil stimulation, which was involved in inflammatory responses. These activities can be explained by functions of active components in rose water such as geraniol and citronellol. These terpenoids are known to penetrate easily through the skin, so we can speculate that cutaneous application of rose water may be sufficient to suppress neutrophil activation and pathogenic invasion of these microbes in the skin. These findings suggest that rose water is a potential candidate for the treatment of superficial infection. Furthermore, as rose water suppressed activation of neutrophils prepared from young females after moderate exercise, rose water may alleviate various types of inflammation through suppression of excessively stimulated neutrophil function.

\section{Anti-inflammatory function of rose water a)}

The study was performed in accordance with the Declaration of Helsinki and was approved by the ethics committee of Teikyo University Review Board. All volunteers gave informed consent to participating in the study.

※Neutrophil adhesion induced by TNF- $\alpha$, LPS and fMLP Human peripheral blood neutrophils obtained from healthy volunteers were incubated with stimulants and rose water for $1 \mathrm{hr}$ at $37^{\circ} \mathrm{C}$ in $5 \% \mathrm{CO}_{2}$. Neutrophil adhesion to the plates was evaluated by measuring the $\mathrm{OD}_{620}$.

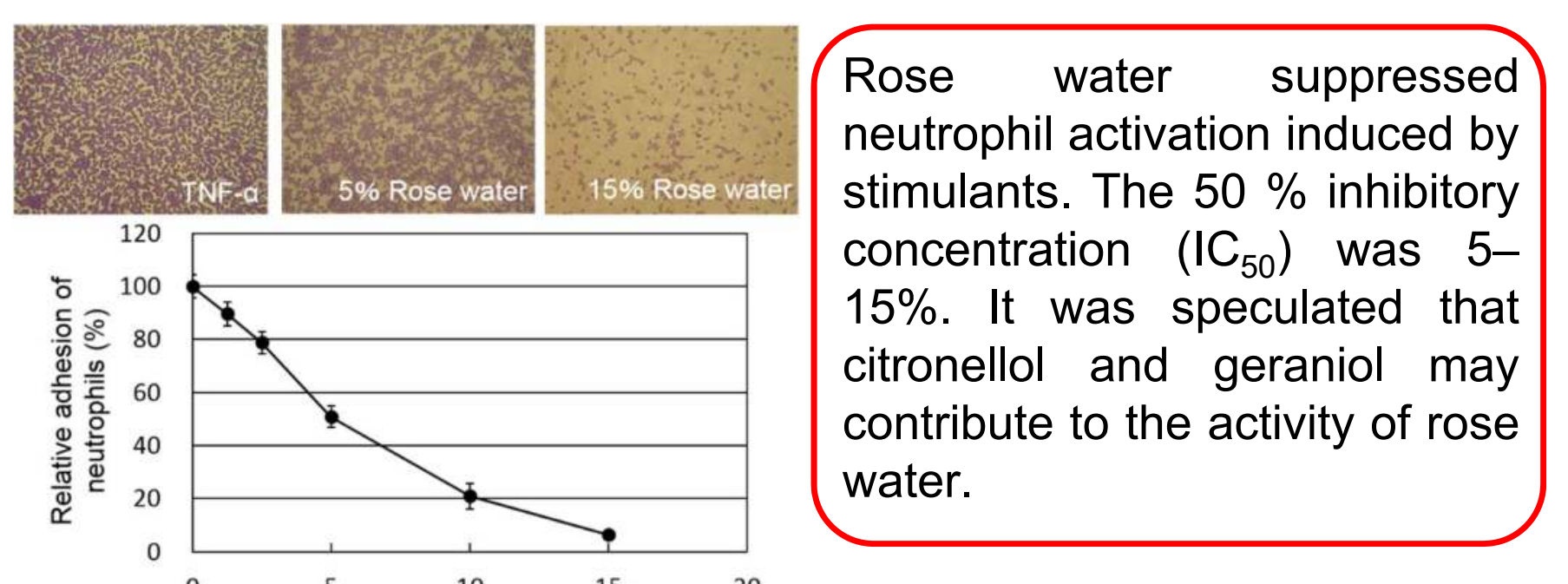

※Expression level of the neutrophil membrane antigen, CD11b, induced by LPS and TNF- $\alpha$ The expression level of CD 11b was measured using a FACSCalibur flow cytometer.

Rose water reduced the LPSand TNF- $\alpha$-induced cell surface expression of CD11b.

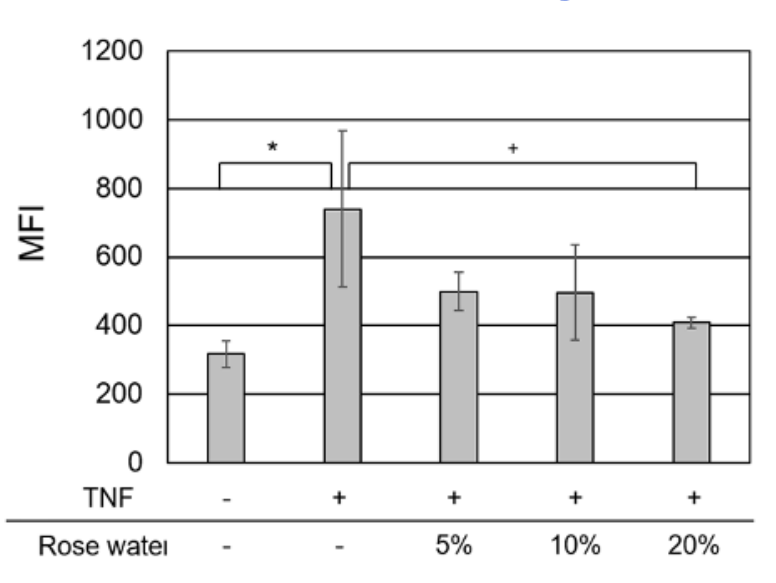

※Neutrophil migration induced by IL-8 and fMLP

Rose water did not affect the migratory capacity of neutrophils with or without chemoattractant.

\section{Anti-inflammatory function after moderate exercise ${ }^{b)}$} Healthy 6 young female volunteers belonging to basketball club run $4.5 \mathrm{~km}$, and then peripheral neutrophils were collected.

Rose water suppressed neutrophil activation induced by TNF- $\alpha$ and $\mathrm{IC}_{50}$ was 3-15\%.

\section{Candida infection and inflammatory symptoms $\sim$ Therapeutic effects of rose water}

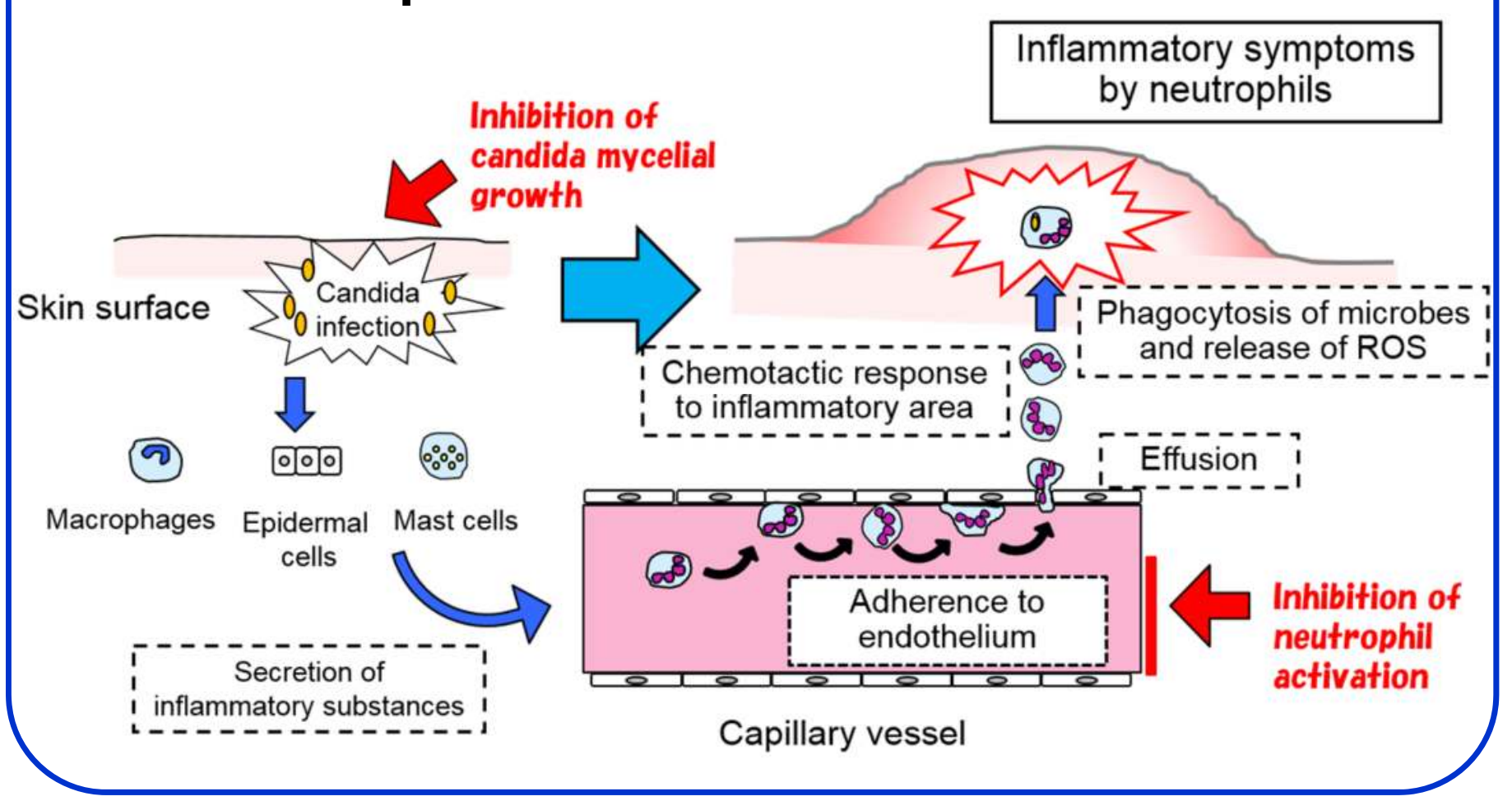

Copyright @ 2018 Naho Maruyama, e-mail: n.maruyama@thu.ac.jp 